Cahiers
de a $\begin{gathered}\text { Recherche } \\ \text { sur les Droits } \\ \text { Fondamentaux }\end{gathered}$

Cahiers de la recherche sur les droits fondamentaux

14 | 2016

Urbanisme et droits fondamentaux

\title{
Expropriation et Convention européenne des droits de l'homme
}

Expropriation and the European Convention on Human Rights

Simon Gilbert

\section{(2) OpenEdition}

\section{Journals}

Édition électronique

URL : https://journals.openedition.org/crdf/591

DOI : $10.4000 /$ crdf.591

ISSN : 2264-1246

Éditeur

Presses universitaires de Caen

Édition imprimée

Date de publication : 1 novembre 2016

Pagination : 73-86

ISBN : 978-2-84133-838-2

ISSN : $1634-8842$

Référence électronique

Simon Gilbert, «Expropriation et Convention européenne des droits de l'homme », Cahiers de la recherche sur les droits fondamentaux [En ligne], 14 | 2016, mis en ligne le 01 octobre 2019, consulté le 14 novembre 2022. URL : http://journals.openedition.org/crdf/591; DOI : https://doi.org/10.4000/crdf. 591

Tous droits réservés 


\title{
Expropriation et Convention européenne des droits de l'homme
}

\author{
Simon GILBERT \\ Professeur de droit public à l'université Paris 12 - Paris-Est-Créteil-Val-de-Marne
}

I. L'existence en droit interne de mécanismes de "cession forcée " assimilables à des " expropriations indirectes » en droit européen

A. Les objets que désigne juridiquement l'expression "cession forcée "

B. Interrogations sur la conventionnalité de certains dispositifs juridiques correspondant à des cessions forcées

II. La conventionnalité du droit de l'expropriation au regard du droit européen
A. La conformité au droit européen des principes structurants de la procédure d'expropriation
B. La conventionnalité des principes relatifs au droit et à l'évaluation de l'indemnité d'expropriation

Le droit français de l'expropriation, dont les origines remontent à l'Ancien Régime ${ }^{1}$, est soumis à une procédure duale depuis 1810, étant écartelé entre deux phases administrative et judiciaire. Si ce modèle «napoléonien » a perduré depuis deux siècles ${ }^{2}$, bien que faisant l'objet d'un nombre d'aménagements considérable, qu'il s'agisse de la fixation des indemnités, des enquêtes publiques, de l'évolution de la notion d'utilité publique, etc., la montée en puissance du droit européen et du droit constitutionnel a conduit à s'interroger sur sa conformité à des standards dont il pouvait sous certains rapports être parfois éloigné. La conventionnalité du droit de l'expropriation est fréquemment mise en cause par les justiciables depuis 1981, date à laquelle ces derniers ont été juridiquement habilités à saisir directement la Cour européenne des droits de l'homme (Cour EDH), notamment en invoquant l'article $6, \S 1$, de la Convention européenne de sauvegarde des droits de l'homme et des libertés fondamentales (Convention EDH), son article 13, relatif au droit au recours effectif, l'article $1^{\text {er }}$ du premier Protocole additionnel à la Convention EDH qui consacre le droit de chacun au « respect de ses biens » $\left(\right.$ al. $\left.1^{\text {er }}\right)$ et stipule que «Nul ne peut être privé de sa propriété que pour cause d'utilité publique et dans les conditions prévues par la loi et les principes généraux du droit international» (al. 2), ainsi que la jurisprudence de la Cour EDH, aujourd'hui incontournable compte tenu de son pouvoir prétorien. L'introduction de la question prioritaire de constitutionnalité (QPC) par la loi constitutionnelle no 2008-724 du 23 juillet 2008 a également fait naître des attentes en matière d'expropriation. Même si celles-ci ont été le plus souvent déçues car, à peu d'exceptions près, les dispositions législatives se sont toujours vu décerner un brevet de constitutionnalité, l'existence de ce dispositif

1. Voir J.-L. Harouel, L'histoire de l'expropriation, Paris, PUF (Que sais-je?), 2000; S. Gilbert, Le juge judiciaire, gardien de la propriété privée immobilière. Étude de droit administratif, Paris, Mare \& Martin, 2011 [issu d'une thèse soutenue en 2006].

2. Voir S. Gilbert, «Permanence et évolution du modèle napoléonien », La semaine juridique, édition administrations et collectivités territoriales, n० 8,21 février 2011, 2078, p. 61-64. 
permet dorénavant aux justiciables de s'interroger sur la conformité du droit de l'expropriation avec, pour horizon, l'espoir qu'il soit réformé afin de respecter davantage les droits et libertés de rang constitutionnel. L'objet de cette étude n'est pas de mettre en perspective la QPC et le recours à la Cour EDH, l'un par rapport à l'autre, pour vérifier si le droit européen, tel qu'interprété, offre ou offrirait plus ou moins de protection aux expropriés que les droits et libertés de rang constitutionnel, tels qu'interprétés par le Conseil constitutionnel. Un tel travail nécessiterait d'être très prudent car il peut exister un écart entre les énoncés et leur interprétation par le Conseil constitutionnel ou la Cour EDH. S'agissant du Conseil constitutionnel, par exemple, l'écart entre le discours idéologique auquel se livre régulièrement ce dernier pour s'ériger en protecteur attentif des droits et libertés de rang constitutionnel, en donnant une certaine représentation de la réalité juridique, et l'effectivité de la protection qu'il assure de ces droits et libertés, est considérable. Notre objectif, en l'espèce plus limité, consiste à dresser un bilan du caractère conventionnel du droit français de l'expropriation ${ }^{3}$ et à identifier, de manière prospective, les possibles risques de condamnation de la France par la Cour EDH pour non-conformité du droit français de l'expropriation. Afin de ne pas limiter notre étude à ce qui correspond stricto sensu à une expropriation en droit français, compte tenu de ce que le droit européen proscrit l'expropriation indirecte qui peut être concrétisée à travers des dispositifs juridiques distincts de l'expropriation en droit interne, nous avons estimé qu'il serait utile d'examiner en premier lieu quels sont, en droit interne, les mécanismes de cession forcée assimilables à des expropriations indirectes en droit européen. Cette question, qui implique de définir ce à quoi correspond une «cession forcée " (ce qui, on le verra, ne relève pas toujours de l'évidence), nous permettra d'identifier des dispositifs juridiques qui permettent d'opérer des cessions forcées de propriété et qui exposent directement la France à des condamnations de la Cour EDH au nom de l'interdiction du recours à l'expropriation indirecte. Après avoir examiné cette question, nous tenterons de mesurer quelle influence a eue et a encore le droit européen dans le cadre de l'écriture du droit de l'expropriation, depuis une trentaine d'années - depuis 1981, plus précisément. Ce sera l'occasion de souligner que si le droit européen déstabilise parfois le droit français de l'expropriation, en ce qu'il conduit la doctrine, les juges administratif et judiciaire ainsi que le législateur et le pouvoir réglementaire à le prendre en compte pour appliquer le droit interne de l'expropriation en le rendant conventionnel ou davantage conventionnel qu'il ne l'est déjà, il est assez rare que la France soit en filigrane invitée à opérer une réforme de ce droit dans la mesure où les condamnations de la Cour EDH en la matière sont très peu nombreuses. L'influence du droit européen ne peut toutefois se limiter aux condamnations de la France. En effet, en déterminant le cas échéant de nouveaux standards, la Cour EDH montre un point d'horizon à atteindre et invite régulièrement les diverses juridictions internes, le législateur ainsi que le pouvoir réglementaire à faire évoluer la procédure d'expropriation, souvent pour se prémunir d'une condamnation. De ce point de vue, l'influence du droit européen sur le droit français de l'expropriation, bien que souvent indirecte, est néanmoins réelle. On examinera en premier lieu l'existence en droit interne de mécanismes de «cession forcée » assimilables à des « expropriations indirectes » en droit européen (I) et en second lieu la conventionnalité du droit français de l'expropriation (II).

\section{L'existence en droit interne de mécanismes de "cession forcée" assimilables à des " expropriations indirectes » en droit européen}

Il convient tout d'abord d'identifier les objets que désigne juridiquement l'expression «cession forcée» (A) puis de faire état des interrogations portant sur la conventionnalité de certains dispositifs juridiques correspondant à des cessions forcées, au regard du droit européen (B).

\section{A. Les objets que désigne juridiquement l'expression "cession forcée "}

La procédure d'expropriation constitue le modèle archétypique de la cession forcée - qui, étant forcée, n'est pas une vente -, même si un transfert amiable de propriété réalisé après la délivrance de la déclaration d'utilité publique (DUP) - et même avant celle-ci - produit les mêmes effets que l'ordonnance d'expropriation, dès lors que le juge de l'expropriation en «donne acte ${ }^{4}$. Sous ce rapport, la préemption ne peut être considérée comme une cession forcée puisque, si le vendeur se trouve privé de la possibilité de choisir l'acheteur, il demeure libre de renoncer au principe de la vente. La cession forcée implique en outre un transfert de propriété. Alors que l'expropriation indirecte, qui constitue un mécanisme ancien consistant à opérer un transfert de propriété forcé sans recourir à

3. Les références doctrinales relatives à ce sujet sont innombrables. Parmi les travaux pionniers: J.-F. Struillou, Protection de la propriété privée immobilière et prérogatives de puissance publique, Paris - Montréal, L'Harmattan, 1996. Plus récemment: R. Hostiou, «Le droit français de l'expropriation et la Convention européenne des droits de l'homme», L'actualité juridique. Droit administratif, 200o, p. 290; R. Hostiou, «La conventionnalité du Code de l'expropriation au regard du droit à un procès équitable. Réflexions à partir du rapport de la Cour de cassation pour l'année 2000 », Revue de droit immobilier, 2002, p. 175; R. Hostiou, «Une tentative de "conventionnalisation" du Code de l'expropriation», en ligne: http://www.pub-law.net/publaw/view.aspx?id=841 (non daté).

4. Art. L. 12-2, al. 2, du Code de l'expropriation puis art. L. 222-2 du nouveau Code de l'expropriation. 
la procédure d'expropriation', a été condamnée en droit interne, à partir des années $1990^{6}$, et l'est, de manière pérenne, en droit européen ${ }^{7}$, la question se pose de savoir si, en admettant l'existence d'une voie de fait ou d'une emprise irrégulière en présence d'une « extinction du droit de propriété», le Tribunal des conflits n'aurait pas, dans les arrêts Bergoend ${ }^{8}$ puis Époux Panizzon ${ }^{9}$, réintroduit, en creux, la possibilité de procéder à une telle expropriation au sens du droit européen.

L'expression «extinction du droit de propriété », bien qu'elle évoque une privation de propriété, a été préférée par le Tribunal des conflits au terme «privation», employé habituellement en matière d'expropriation (art. 17 de la Déclaration des droits de l'homme et du citoyen de 1789). Comment l'expliquer? Probablement en raison de son indétermination sémantique relative, afin que les requérants, ne sachant pas précisément quels objets juridiques elle désigne, renoncent progressivement à s'en prévaloir, ceci à fin de restreindre sinon de mettre un terme à la voie de fait ainsi qu'à l'emprise irrégulière. Certes, en définissant une des formes de la voie de fait ${ }^{10}$ et de l'emprise irrégulière ${ }^{11}$ à partir du critère de l'extinction du droit de propriété $^{12}$, le Tribunal des conflits a probablement souhaité - peut-être était-ce même son principal objectif? - distinguer les concepts juridiques de voie de fait et d'emprise irrégulière de celui d'expropriation, bien qu'il n'ait pas jugé utile d'indiquer, fût-ce même de manière implicite, les types d'atteintes que désigne l'extinction du droit de propriété. Toujours est-il que le terme « extinction » revêt, dans le langage juridique usuel, une signification proche sinon synonyme de celui de "privation "; il est question dans les deux cas de ne plus permettre au propriétaire victime de l'atteinte de continuer d'exercer l'ensemble des droits d'un propriétaire sur le bien objet de l'atteinte et, par conséquent, de le priver de sa propriété. Le recours à l'expression «extinction du droit de propriété» laisse donc des interrogations en suspens s'agissant de sa signification, mais il en laisse aussi au regard de l'office du juge de la voie de fait: comment ce dernier pourrait-il, en effet, faire cesser une atteinte extinctive du droit de propriété si ledit droit a été "éteint»? Mutatis mutandis, observons que si, en matière d'expropriation, l'extinction des droits réels et personnels par l'ordonnance (art. L. 12-2 de l'ancien Code de l'expropriation; art. L. 222-2 du nouveau Code) ne permet pas à l'ancien propriétaire exerçant une action en rétrocession de recouvrer ces droits, c'est précisément parce qu'ils ont été éteints.

La terminologie employée par le Tribunal des conflits est donc imprécise en ce que ce dernier n'a pas désigné les atteintes que vise l'expression «extinction du droit de propriété». Mais elle est, de plus, confuse, dans la mesure où ce dernier emploie de manière interchangeable les expressions «extinction du droit de propriété » et "dépossession définitive», alors que, traditionnellement, la «dépossession", qu'elle soit ou non définitive, s'oppose à la "privation de propriété» et devrait, sous ce rapport, être distinguée de l'«extinction du droit de propriété» qui s'apparente à une "privation». En effet, dans la décision Époux Panizzon, précitée, le tribunal répartiteur de compétences décide que :

[...] si l'occupation de la parcelle de terrain appartenant à M. et $\mathrm{M}^{\mathrm{me}} \mathrm{X}$... par la commune de Saint-Palais-sur-Mer a porté atteinte au libre exercice de leur droit de propriété sur ce bien, elle n'a pas eu pour effet de les en déposséder définitivement $[\ldots]$.

Cette confusion conceptuelle s'observe également dans un arrêt de la cour administrative d'appel de Marseille

5. L'expropriation indirecte, dont l'appellation est d'origine doctrinale (selon A. Texier, De l'expropriation indirecte, thèse de doctorat en droit, Paris, 1905, p. 11, l'expression "expropriation indirecte» a été imaginée par la doctrine qui emploie les termes d' "expropriation administrative», d'«expropriation tacite» ou d'«expropriation irrégulière»), fait son apparition sous la Restauration. Elle repose sur l'idée que, dans l'hypothèse où une expropriation est réalisée sans recours à la procédure d'expropriation, les tribunaux doivent être autorisés à intervenir pour indemniser le propriétaire, compte tenu du fait qu'ils seraient normalement intervenus si la procédure avait été respectée (voir M. Hauriou, Précis de droit administratif et de droit public, $3^{\mathrm{e}}$ éd., Paris, L. Larose, 1897, p. 757 : «l'expropriation pour cause d'utilité publique n'étant au fond qu'une dépossession définitive avec transfert, entourée d'une certaine procédure protectrice, et les tribunaux étant compétents, ils doivent l'être $a$ fortiori lorsqu'il y a dépossession définitive et transfert de propriété sans procédure ou avec des procédures moins protectrices»). André Vouters donne une intéressante explication du phénomène de l'expropriation indirecte qui vise à montrer que c'est le droit applicable qui a participé à l'émergence de la «théorie» de l'expropriation indirecte. C'est, indique l'auteur, en «raison de la générosité excessive manifestée par le jury d'expropriation» - que l'on retrouve déjà dans l'indemnisation prévue dans le cadre des lois de 1810 et 1833-, qu'il «pouvait être tentant pour les entrepreneurs de travaux publics, en vue d'éviter le paiement de telles indemnités, d'envahir purement et simplement les terrains nécessaires à l'exécution des travaux entrepris, ce qui, en l'absence de la théorie de l'expropriation indirecte, les aurait amenés devant le Conseil de Préfecture, juridiction vis-à-vis de laquelle le paiement de l'indemnité, même tenant compte de la responsabilité encourue par l'entrepreneur, aurait pu être finalement moins élevé» (De la règle que l'autorité judiciaire est gardienne de la propriété privée. Essai historique et critique, thèse de doctorat en droit, Lille, Douriez-Bataille, 1940, p. 213).

6. Cass., Ass. plén., 6 janvier 1994, Consorts Baudon de Mony c. Électricité de France, pourvoi no 89-17049: «le transfert de propriété, non demandé par le propriétaire, ne peut intervenir qu'à la suite d'une procédure régulière d'expropriation "; Cahiers juridiques de l'électricité et du gaz, n 501 , août 1994, p. 413, concl. M. Jéol et note M. Renard-Payen. CE, 19 avril 1991, Époux Denard: Revue française de droit administratif, 1992, p. 63.

7. Cour EDH, 30 mai 200o, Belvedere Alberghiera Srl c. Italie, $\mathrm{n}^{\circ}$ 31524/96; Cour EDH, 16 avril 2013, Rolim Comercial SA c. Portugal, $\mathrm{n}^{\circ}$ 16153/o9.

8. TC, 17 juin 2013, Bergoend c. Société ERDF Annecy Léman, $\mathrm{n}^{\circ}$ 3911: Revue française de droit administratif, 2013, p. 1041, note P. Delvolvé; Droit administratif, 2013, p. 49-52, comm. no 86 , note S. Gilbert.

9. TC, 9 décembre 2013, Époux Panizzon, $\mathrm{n}^{\circ} 3931$ : Droit administratif, 2014, p. 40, comm. n² 25, note S. Gilbert.

10. La voie de fait, telle que définie par l'arrêt Bergoend, précité, est également caractérisée par une atteinte à une «liberté individuelle».

11. Le juge administratif est désormais compétent pour indemniser et faire cesser une emprise irrégulière "non extinctive du droit de propriété» (TC, 9 décembre 2013, Époux Panizzon).

12. Nous écrivons «une des formes» car le champ de la voie de fait porte aussi sur la «liberté individuelle» et qu'il existe désormais des emprises irrégulières non extinctives du droit de propriété. 
ayant jugé, à propos du broyage d'arbres truffiers, qu'il existait une extinction du droit de propriété révélatrice d'une emprise irrégulière dans la mesure où l'atteinte entraînait une "disparition définitive», une "dépossession définitive $»^{13}$ desdits arbres. Dans un arrêt de la cour administrative d'appel de Bordeaux, il a été déduit notamment de la non-utilisation de la procédure d'expropriation qu'il n'y avait pas d'extinction du droit de propriété, ce qui confirme la difficulté qu'éprouve le juge administratif à mettre en œuvre ce critère ${ }^{14}$.

Si le critère de l'extinction du droit de propriété était entendu, au titre de la voie de fait ou de l'emprise irrégulière, comme étant associé à un possible transfert de propriété ou à une "privation de propriété», cela poserait, nous semble-t-il, un problème de conformité juridique au regard du droit européen, dans la mesure où la jurisprudence de la Cour EDH interdit le recours à l'expropriation indirecte. Est-ce le cas? Cela n'est pas impossible compte tenu de la façon dont a été mis en œuvre jusqu'à aujourd'hui le critère de l'extinction du droit de propriété. Si, en effet, la dépossession est définitive ou s'il y a destruction du bien, la frontière avec la privation de propriété, c'est-à-dire l'expropriation indirecte, au sens du droit européen, risque d'être difficile à tracer. Dans tous les cas, pour qu'il y ait une expropriation au sens du droit européen, rappelons que les victimes de l'atteinte doivent être propriétaires du bien concerné, ce que la Cour EDH a eu l'occasion de préciser notamment dans l'affaire Époux Depalle ${ }^{15}$, à propos de la destruction d'une maison édifiée sur le domaine public grâce une autorisation d'occupation temporaire précaire et révocable ayant cessé de produire ses effets ${ }^{16}$; de ce point de vue, il convient de distinguer le « droit au respect des biens » (art. $1^{\text {er }}$ du premier Protocole additionnel à la Convention $\mathrm{EDH}$ ), qui n'implique pas l'existence d'un titre de propriété ${ }^{17}$ pour que l'atteinte soit constituée, et l'expropriation, directe ou indirecte, qui implique que la victime est bien le propriétaire du bien objet de l'atteinte. Après avoir examiné le contenu du concept de «cession forcée», qui laisse quelques interrogations en suspens, il convient de présenter les divers dispositifs juridiques existant en droit interne et qui, sans correspondre en droit interne à une expropriation, pourraient être qualifiés d'expropriation indirecte en droit européen et conduire, de ce fait, à une condamnation de la France par la Cour EDH, dans la mesure où l'expropriation indirecte est interdite en droit européen (voir supra).

\section{B. Interrogations sur la conventionnalité de certains dispositifs juridiques correspondant à des cessions forcées}

Certains mécanismes juridiques conduisent à des cessions forcées de propriété qui sont susceptibles d'être assimilés à une expropriation indirecte en droit européen, bien que le recours à ce procédé soit interdit par le droit européen tel qu'interprété par la Cour EDH. Quels sont ces mécanismes juridiques?

Le régime juridique de l'alignement, pour commencer $^{18}$, pose question du point de vue de sa conventionnalité au regard de l'article $1^{\text {er }}$ du premier Protocole additionnel à la Convention EDH, car le plan d'alignement a pour effet de transférer la propriété privée au profit d'une personne publique dès lors que le terrain est nu. Bien que le Conseil constitutionnel ait décidé que «l'alignement n'entre pas dans le champ d'application de l'article 17 de la Déclaration de 1789 " au motif qu'il

[...] n'attribue à la collectivité publique le sol des propriétés qu'il délimite que dans le cadre de rectifications mineures du tracé de la voie publique [...], ne permet ni d'importants élargissements ni a fortiori l'ouverture de voies nouvelles [et] qu'il ne peut en résulter une atteinte importante à l'immeuble $[\ldots]^{19}$

il apparaît que l'usage de ce dispositif, tel que prévu aux articles L. 112-1 et L. 112-2 du Code de la voirie, constitue bien une cession forcée de propriété. On note d'ailleurs que c'est l'existence d'une «cession forcée de propriété privée » constituée par la publication du plan d'alignement établi unilatéralement, «sans que soit constatée sa nécessité publique ni qu'il soit fait droit à une indemnisation préalable $»^{20}$, que les requérants avaient dénoncée, dans la QPC transmise au Conseil constitutionnel par le Conseil d'État. Les alinéas 1, 2 et 3 de l'article L. 112-2 du Code de la voirie évoquent d'ailleurs sans ambiguïté un tel transfert de propriété par l'Administration, de manière unilatérale et non amiable:

13. CAA Marseille, $2^{\mathrm{e}}$ ch., 5 juin 2014, Monsieur D., $\mathrm{n}^{\mathrm{o}} 12 \mathrm{MAoo} 144$

14. CAA Bordeaux, 13 novembre 2014, Madame A., $\mathrm{n}^{\circ}$ 13BXoo121: «qu'en l'absence d'intervention d'un accord amiable avec cette dernière ou d'institution d'une servitude dans les conditions prévues par l'article 12 de la loi du 15 juin 1906 sur les distributions d'énergie ou encore de l'accomplissement d'une procédure d'expropriation pour cause d'utilité publique, le tribunal administratif était compétent pour connaître des conclusions tendant à la réparation des conséquences dommageables de cette situation, même si la société Électricité de France est une société de droit privé en application des dispositions de l'article 24 de la loi du 9 août 2004; que par suite, l'exception d'incompétence soulevée par la société EDF, et non expressément abandonnée, doit être écartée».

15. Cour EDH, GC, Époux Depalle c. France, 29 mars 2010, nº 34044/o2.

16. Rappelons que dans cette affaire, le non-renouvellement d'une autorisation d'occupation privative du domaine public et l'injonction faite au requérant de détruire la maison achetée régulièrement, en 1960, peuvent, selon la Cour EDH, s'analyser «en une réglementation de l'usage des biens» et non en une "privation de propriété». Voir également, le même jour, Cour EDH, 29 mars 2010, Brosset-Triboulet et autres c. France, $\mathrm{n}^{\mathrm{o}} 34078 / 02$ : L'actualité juridique. Droit administratif, 2010, p. 647.

17. Cour EDH, 30 novembre 2004, Öneryildiz c. Turquie, no 48939/99: L'actualité juridique. Droit administratif, 2005, p. 1133 sq., note S. Rabiller.

18. On examinera divers dispositifs susceptibles de constituer une cession forcée, sans qu'un ordre particulier de présentation s'impose à ce titre.

19. CC, déc. $\mathrm{n}^{\mathrm{o}}$ 2011-201 QPC du 2 décembre 2011, Consorts D., cons. 5.

20. Ibid., cons. 3 . 
[al. 1] La publication d'un plan d'alignement attribue de plein droit à la collectivité propriétaire de la voie publique le sol des propriétés non bâties dans les limites qu’il détermine.

[al. 2] Le sol des propriétés bâties à la date de publication du plan d'alignement est attribué à la collectivité propriétaire de la voie dès la destruction du bâtiment.

[al. 3] Lors du transfert de propriété, l'indemnité est, à défaut d'accord amiable, fixée et payée comme en matière d'expropriation.

Il est dès lors difficile de comprendre comment le Conseil constitutionnel a pu de manière juridiquement conforme considérer que l'alignement n'avait pas d'effets comparables à ceux d'une expropriation. Sans s'interroger plus précisément sur la constitutionnalité de ce dispositif, la question se pose de savoir si, du point de vue du droit européen, ce dispositif risque d'entraîner un jour une condamnation de la France? L'article $1^{\text {er }}$ du premier Protocole additionnel dispose à son alinéa 2 que «Nul ne peut être privé de sa propriété que pour cause d'utilité publique et dans les conditions prévues par la loi et les principes généraux du droit international ». Dès lors que les articles L. 112-1 et L. 112-2 du Code de la voirie prévoient un transfert de propriété inhérent à l'alignement, il existe certes une «loi » au sens de l'article susvisé. Mais, s'agissant de la «cause d'utilité publique» évoquée par le même article du Protocole, le fait qu'il existe une «enquête publique» visant à évaluer l'utilité publique dudit plan, en amont de son édiction, sans déclaration d'utilité publique qui puisse faire l'objet d'un contrôle juridictionnel, conduit à penser qu'il est possible que la Cour EDH, si elle était saisie, considère ledit dispositif comme étant insuffisant pour se conformer à l'article $1^{\text {er }}$ du premier Protocole additionnel à la Convention EDH au motif qu'un tel dispositif s'apparente à une expropriation indirecte sans correspondre ni même présenter les garanties d'une véritable expropriation.

La cession gratuite de terrain représente un autre dispositif juridique dont la conformité au droit européen interroge en ce qu'elle est susceptible de s'apparenter à une expropriation indirecte. Il s'agit d'un mécanisme prévu par le Code de l'urbanisme (art. L. 332-6-1, $2^{\circ}, \mathrm{e}^{21}$ ) en vertu duquel le bénéficiaire d'une autorisation d'urbanisme peut être contraint de céder à l'Administration une portion du terrain d'assiette du projet autorisé à certaines conditions mais sans indemnisation; c'est pour cette raison que la cession est qualifiée de "gratuite». Certes, le Conseil d'État a considéré ce dispositif comme étant compatible avec le «droit au respect des biens» inscrit à l'article $1^{\mathrm{er}}$ du premier Protocole additionnel à la Convention $\mathrm{EDH}^{22}$, estimant que, dès lors que l'obligation de céder gratuitement une portion de terrain ne représente pas plus de $10 \%$ de la superficie du terrain concerné, il ne saurait être question de privation de propriété, comme en matière d'expropriation $^{23}$. Mais, dans un registre distinct, l'assemblée plénière de la Cour de cassation a jugé sérieuse la question de la conformité de cette disposition du Code de l'urbanisme au droit de propriété, garanti par les articles 2 et 17 de la Déclaration de 1789, ainsi qu'au principe d'égalité devant les charges publiques, garanti par l'article 13 du même texte ${ }^{24}$ et a transmis au Conseil constitutionnel une QPC y afférant. Étudiant ladite QPC, le Conseil constitutionnel ${ }^{25}$ a invalidé (cons. 4) le point e du $2^{\circ}$ de l'article L. 332-6-1 du Code de l'urbanisme, précité, au motif que la disposition «ne définit pas les usages publics auxquels doivent être affectés les terrains» et que le législateur aurait dû déterminer le régime de la propriété sur le fondement de l'article 34 de la Constitution, signe que ce dernier avait fait preuve d'une incompétence négative. En creux, lesdites dispositions «litigieuses » n'ont pas été invalidées au motif que la cession serait gratuite et qu'elle porterait à ce titre atteinte à l'article 17 de la Déclaration de 1789 . Si cette décision méconnaît, nous semble-t-il, les dispositions de la Déclaration de 1789 , la conventionnalité dudit dispositif se pose au regard de l'article $1^{\text {er }}$ du premier Protocole additionnel à la Convention EDH. Il semble juridiquement discutable de réduire cette "cession gratuite», comme l'a décidé le Conseil d'État dans l'arrêt Schiocchet, précité, à une «réglementation de l'usage des biens » donnant lieu à une indemnisation uniquement en cas de disproportion entre les causes et les conséquences d'une telle réglementation. Car la «cession» en question s'apparente à une véritable privation de propriété «hors expropriation», ce que la doctrine n'a pas manqué de souligner ${ }^{26}$. Nous pensons qu'une telle cession gratuite est contraire à l'article $1^{\text {er }} \mathrm{du}$ premier Protocole additionnel à la Convention EDH qui, tel qu'interprété par la Cour EDH, exige que toute privation de propriété soit indemnisée, sauf circonstances exceptionnelles ${ }^{27}$. Dès lors, à moins d'identifier de telles circonstances exceptionnelles, ce qui semble très difficile si l'on s'en

21. L'article prévoit la possibilité pour les communes de contraindre les constructeurs, par une prescription incluse dans l'autorisation d'occupation du sol, de leur céder gratuitement une partie de leur terrain.

22. CE, 11 février 2004, Schiocchet, $\mathrm{n}^{\circ} 211510$.

23. Ibid.: «Les dispositions précitées des articles L. 332-6 et L. 332-6-1 du Code de l'urbanisme, en ce qu'elles permettent de subordonner la délivrance d'un permis de construire à la cession gratuite de $10 \%$ au plus de la superficie du terrain au titre de la contribution du bénéficiaire aux dépenses d'équipements publics, ont pour objet non pas, comme la procédure d'expropriation pour cause d'utilité publique, de priver une personne de la propriété d'un bien mais de réglementer le droit de construire, qui, faute d'être un droit nécessairement attaché à la propriété du terrain, relève de l'usage d'un tel bien au sens des stipulations précitées du protocole».

24. Cass., Ass. plén., 25 juin 2010, Société Esso SAF, nº 10-40.008.

25. CC, déc. $\mathrm{n}^{\circ}$ 2010-33 QPC du 22 septembre 2010, Société Esso SAF.

26. R. Béraud, «Une expropriation qui n’ose pas dire son nom : la cession gratuite de 10 \% », Annales des loyers, 1981, p. 870; E. Carpentier, «La QPC sur la cession gratuite de terrain ou la suite du dialogue de sourds entre les juridictions françaises», Recueil Dalloz, 2011, p. 136.

27. Cour EDH, 21 février 1986, James et autres c. Royaume-Uni, nº 8793/79, \$ 34; Cour EDH, 9 décembre 1994, Les saints monastères c. Grèce, $\mathrm{n}^{\circ} 13092 / 87, \S 71$. 
tient aux objets qui ont reçu une telle qualification par la Cour $\mathrm{EDH}^{28}$, une sanction de la France semble probable ${ }^{29}$.

En troisième lieu, le transfert d'office sans indemnité au domaine public d'une voie privée ouverte à la circulation publique dans des ensembles d'habitation nous semble susceptible d'être considéré comme non conforme au regard du droit européen. À quoi correspond ce «transfert d'office»? Aux termes de l'article L. 318-3 du Code de l'urbanisme:

La propriété des voies privées ouvertes à la circulation publique dans des ensembles d'habitations peut, après enquête publique [...], être transférée d'office sans indemnité dans le domaine public de la commune sur le territoire de laquelle ces voies sont situées. La décision de l'autorité administrative portant transfert vaut classement dans le domaine public et éteint, par elle-même et à sa date, tous droits réels et personnels existant sur les biens transférés.

Certes, le Conseil constitutionnel a reconnu comme étant valides de telles dispositions législatives, estimant qu'il n'était ainsi pas porté atteinte à l'article 17 de la Déclaration de $1789^{30}$. La motivation de ladite décision mérite d'être rappelée; il est décidé

[...] qu'un tel transfert est conditionné, sous le contrôle du juge administratif, par l'ouverture à la circulation générale de ces voies, laquelle résulte de la volonté exclusive de leur propriétaire d'accepter l'usage public de son bien et de renoncer par là à son usage purement privé; que le législateur a entendu en tirer les conséquences en permettant à l'autorité administrative de conférer à ces voies privées ouvertes à la circulation publique un statut juridique conforme à leur usage $[\ldots]^{31}$.

Ce raisonnement est particulièrement contestable car, outre le fait qu'il est manifestement contraire à l'article 17 de la Déclaration de 1789 en tant qu'il postule que l'acceptation d'un usage public d'un bien privé équivaut, de la part de son propriétaire, à un renoncement à son usage purement privé et qu'il admet une privation de propriété sans indemnité, sa conformité à l'article $1^{\text {er }}$ du premier Protocole additionnel à la Convention EDH pose question pour les mêmes raisons que celles invoquées à l'égard du dispositif précité relatif à la cession gratuite.
La conventionnalité du régime juridique relatif à l'incorporation des propriétés privées submergées dans le domaine public maritime naturel pose également question. L'article L. 2111-4, $1^{\circ}$ du Code général de la propriété des personnes publiques permet un transfert gratuit de propriété au profit de l'État, au détriment du propriétaire, du fonds submergé par les plus hauts flots qui devient un rivage de la mer ${ }^{32}$. Le droit de propriété privée disparaît au profit du domaine public de l'État lorsque le fonds est recouvert par les plus hauts flots, en l'absence de perturbations météorologiques exceptionnelles. Le Conseil constitutionnel a estimé ce dispositif conforme à la Déclaration de 1789, au motif qu'il n'était question que d'un transfert de propriété conditionné par un «critère physique objectif indépendant de la volonté de la puissance publique» - comme si la norme juridique et ses effets étaient indépendants de la volonté du législateur ${ }^{33}$ - et que les dispositions contestées n'entraînent dès lors ni une privation de propriété au sens de l'article 17 de la Déclaration de 1789 , ni une atteinte contraire à l'article 2 de la Déclaration de $1789^{34}$. Dans la mesure où ce mécanisme conduit à un transfert de propriété privée au profit du domaine public de l'État sans indemnisation, sa conventionnalité pose de toute évidence question au regard des exigences du droit au respect des biens tel qu'interprété par la Cour EDH.

Il existe ainsi en droit interne une série de dispositifs juridiques qui, parce qu'ils conduisent à une cession forcée, le cas échéant sans indemnité, ne semblent pas conformes au droit européen et exposent potentiellement la France à une condamnation au titre de l'expropriation indirecte. Examinons maintenant dans quelle mesure le droit français de l'expropriation se conforme au droit européen.

\section{La conventionnalité du droit de l'expropriation au regard du droit européen}

Le droit français de l'expropriation est aujourd'hui confronté à la Convention EDH (notamment l'art. 6,

28. Cour EDH, GC, 30 juin 2005, Jahn et autres c. Allemagne, $\mathrm{n}^{\circ}$ 46720/99, $\$ 117$ : «la réunification allemande»; Cour EDH, 12 novembre 2002, Zvolsky et Zvolská c. République tchèque, $\mathrm{n}^{\circ}$ 46129/99, $\$ 72$ : «l'acquisition de biens sans indemnité sous des régimes dits socialistes ».

29. La Cour EDH a, à diverses reprises, jugé que l'absence de toute indemnisation rompt le juste équilibre entre les exigences de l'intérêt général et les impératifs de la sauvegarde des droits individuels. Voir, par exemple, Cour EDH, 8 juillet 2008, Turgut et autres $c$. Turquie, $\mathrm{n}^{\circ} 1411 / 03$, $\$ 92$ : «en cas de privation de propriété, [...] une absence totale d'indemnisation ne saurait se justifier sur le terrain de l'article 1 du Protocole n ${ }^{\circ} 1$ que dans des circonstances exceptionnelles »; Cour EDH, 5 avril 2007, Nastou c. Grèce (no 2), n 16163/02, \$33; Cour EDH, GC, 30 juin 2005 , Jahn et autres c. Allemagne, nº 46720/99, 72203/o1 et 72552/o1, \$111; Cour EDH, 9 décembre 1994, Les saints monastères c. Grèce, précité, $\$ 71$. En l'espèce, les requérants n'ont reçu aucune indemnité pour le transfert de leur bien au Trésor public, par application de l'article 169, \$2, de la Constitution. La Cour note que le gouvernement n'a invoqué "aucune circonstance exceptionnelle pour justifier l'absence totale d'indemnisation".

30. CC, QPC n 2010-43 du 6 octobre 2010, Époux A.

31. Ibid., cons, 4

32. Cette disposition codifie la jurisprudence du Conseil d'État (CE, 17 octobre 1934, Dupont, Recueil Lebon, p. 927).

33. En imposant d'adopter tel ou tel comportement, le droit ne décrit pas le réel mais le constitue; c'est un effet performatif. La norme nous impose de concevoir le « réel » de telle ou telle manière; il y a, à propos de l'exemple évoqué dans le corps du texte, juridiquement un transfert de propriété non pas en raison du mouvement des flots mais parce que le système juridique l'a prévu. Le Conseil constitutionnel ne prend pas pleinement la mesure de ce que le réel ne prescrit pas, n'est pas normatif, ne parle pas à l'impératif; c'est l'enjeu de la «loi de Hume» (Enquête sur l'entendement humain, 1748) selon laquelle les normes ne peuvent être inférées des faits car on ne peut logiquement faire dériver de façon valide un énoncé normatif sur la base de données qui ne sont pas, elles-mêmes, normatives.

34. CC, déc. n 2013-316 QPC du 24 mai 2013, SCI Pascal et autre. 
$\$ 1$ ), aux dispositions de l'article $1^{\text {er }}$ du premier Protocole additionnel:

Toute personne physique ou morale a droit au respect de ses biens.

Nul ne peut être privé de sa propriété que pour cause d'utilité publique et dans les conditions prévues par la loi et les principes généraux du droit international.

ainsi qu'à la jurisprudence de la Cour EDH, aujourd'hui incontournable, ce qui a pour effet de le déstabiliser et, le cas échéant, de conduire à ce qu'il soit réformé. Dresser le bilan de cette influence oblige toutefois à observer son caractère assez limité sur le droit dispositionnel et jurisprudentiel interne. Est symptomatique, à cet égard, le fait que très peu d'affaires emblématiques relatives au droit français de l'expropriation ont été portées devant la Cour EDH et ont abouti à une condamnation de la France - on cite à ce titre à peine quelques décisions: Guillemin ${ }^{35}$, Lallement ${ }^{36}$, Motais de Narbonne ${ }^{37}$, Yvon ${ }^{38}$ - alors que c'est en 1981 qu'a été reconnu aux Français le droit d'exercer un recours devant la Cour EDH après épuisement des voies de recours internes. Plusieurs aspects de la procédure ont fait l'objet de réformes mais, compte tenu des différences de cultures qui caractérisent le droit français de l'expropriation et le droit européen, et, au sein de celui-ci, plus précisément le «droit au respect des biens », entendu au sens du premier Protocole additionnel à la Convention $\mathrm{EDH}$, on aurait pu s'attendre à ce qu'il y ait davantage de condamnations de la France à ce titre, le droit de l'expropriation étant notamment marqué par un fort imperium étatique qui, sous de nombreux rapports, n'apparaît pas pleinement conforme avec le droit européen d'inspiration plus libéral ${ }^{39}$. Pour examiner maintenant les aspects du droit de l'expropriation dont la conventionnalité a été ou pourrait être remise en question, on distinguera les aspects structurants de la procédure (A) des principes relatifs au droit et à l'évaluation de l'indemnité (B).

\section{A. La conformité au droit européen des principes structurants de la procédure d'expropriation}

Divers aspects de la procédure d'expropriation ont été remis en question au regard des exigences du droit européen. Sous ce rapport, il est certain que le droit français de l'expropriation a été en partie déstabilisé. Pour autant, il serait exagéré d'affirmer qu'on l'a profondément réformé pour le mettre en conformité avec le droit européen si l'on recense les aspects de la procédure qui ont été modifiés à la suite de condamnations de la France par la Cour EDH. Indépendamment de telles condamnations, il est indéniable qu'un certain nombre de modifications ont été apportées à la procédure d'expropriation, ce qui atteste que l'influence du droit européen sur ladite procédure, fût-ce de manière indirecte, est réelle.

Une des condamnations de la France les plus emblématiques en raison du caractère non conventionnel du droit français de l'expropriation a concerné le commissaire du gouvernement. Le statut du commissaire du gouvernement a en effet été remis en question par la Cour EDH dans l'arrêt Yvon (précité), au motif que le commissaire bénéficiait d'un accès privilégié au fichier immobilier, ce qui rompait le principe d'égalité des armes. Cette condamnation a conduit le législateur à modifier l'article L. 135 B du Livre des procédures fiscales, le 13 juillet 2006 (loi n ${ }^{\circ}$ 2006-872, art. 21), afin que l'accès équitable aux valeurs foncières soit garanti ${ }^{40}$. Cette caractéristique du droit de l'expropriation remise en cause était éminemment «napoléonienne» dans l'esprit. Au XIX siècle, à

35. Cour EDH, 21 février 1997, Guillemin c. France, no 105/1995/611/699: L'actualité juridique. Droit administratif, 1997, p. 399, note R. Hostiou.

36. Cour EDH, 11 avril 2002, Lallement c. France, no 46044/99: L'actualité juridique. Droit administratif, 2002, p. 686, note R. Hostiou.

37. Cour EDH, 2 juillet 2002, Motais de Narbonne c. France, $\mathrm{n}^{\circ}$ 48161/99: L'actualité juridique. Droit administratif, 2002, p. 1226, note R. Hostiou.

38. Cour EDH, 24 avril 2003, Yvon c. France, n 44962/98: Recueil Dalloz, 2003, p. 2456, note R. Hostiou; L'actualité juridique. Droit administratif, 2003, p. 869; L'actualité juridique. Droit immobilier, 2003, p. 361, note D. Musso.

39. La plupart des réformes du droit de l'expropriation menées jusqu'aux années 2000 ont été animées par la volonté de renforcer la puissance publique et de restreindre les garanties des propriétaires expropriés. C'est notamment la crainte de voir son autorité menacée qui, en 1810, inspire à Napoléon la désignation du juge judiciaire pour prononcer le transfert de propriété: «[...] en s’accoutumant à jouer avec la propriété on la viole, et [...] il en résulte des abus révoltants qui mécontentent l'opinion publique» (cité par C. Durand, «Le régime juridique de l'expropriation pour utilité publique sous le Consulat et le Premier Empire», Annales de la faculté de droit d'Aix-en-Provence, 1948, p. 5-104, ici p. 104; nous soulignons). C'est également le besoin de protéger le Trésor public des évaluations très élevées des indemnités d'expropriations effectuées par les conseils de préfecture qui l'a convaincu de mener la réforme de 1810 (le rapport de la loi du 8 mars 1810 relève que la mise en œuvre de la loi de 1807 relative au dessèchement des marais n'a pas été favorable au Trésor public tant les indemnités attribuées aux propriétaires expropriés se sont révélées prohibitives pour l'État: «de trop nombreux exemples d'estimation scandaleusement préjudiciables au trésor public», rappelle le comte Berlier, «se présentaient pour que le législateur ne dût pas se tenir en garde contre de tels abus: s'il est dû beaucoup de faveur au propriétaire, ce ne doit jamais être aux dépens de la justice, ni en lésant l'État»; voir l'exposé des motifs du projet de loi par le comte Berlier, Recueil Sirey, 1810, 2, 103-106). De plus, lorsque l'on réforme la loi de 1810, c'est parce que les indemnités accordées par le juge judiciaire sont trop élevées et que la procédure est jugée trop lente: on attend du jury créé en 1833 , formé de propriétaires - aussi contribuables -, qu'il détermine des indemnités plus basses qu'auparavant en se comportant plus en contribuables qu'en propriétaires (ce ne sera pas le cas). La réforme de 1841 est quant à elle animée par le désir d'exproprier plus vite: on introduit donc l'envoi en possession provisoire en créant une procédure d'urgence. Lorsque, en 1914 et en 1918, on modifie la composition du jury en introduisant notamment des fonctionnaires dont le directeur du service des Domaines ou son délégué, c'est de nouveau, et de façon assumée, pour réduire les indemnités d'expropriation. Quand, en 1935, on crée la commission arbitrale d'évaluation des indemnités, c'est toujours pour réduire les indemnités demeurées trop élevées aux yeux du gouvernement. Il n’y a dès lors plus de jury souverain, mais une commission, présidée par un magistrat et composée notamment de deux fonctionnaires, qui détermine le montant de l'indemnité. Quant à la réforme de 1962, qui modifie les règles d'évaluation en imposant l'évaluation de la valeur des biens sur la base de leur valeur un an avant l'ouverture de l'enquête préalable, elle est à nouveau déterminée par la préoccupation de défendre le Trésor public (loi nº 62-848 du 26 juillet 1962, art. 22).

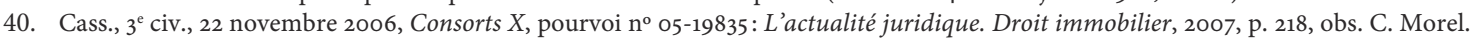


l'époque où l'indemnité d'expropriation était fixée par un jury, l'Administration, en l'absence de commissaire du gouvernement, tentait déjà d'exercer des pressions pour que l'indemnité d'expropriation fût déterminée de manière favorable à la puissance publique. Théophile Ducrocq observait en 1866, dans un article intitulé «Des expropriants, du droit de poursuite appartenant à chacun d'eux, et de la place qu'ils doivent occuper à l'audience du jury d'expropriation ${ }^{41}$, s'agissant de la place occupée par l'expropriant à l'audience du jury d'expropriation chargé de fixer l'indemnité, que les expropriants étaient accoutumés à occuper «le siège du ministère public» au sein des cours d'assises, laissant le plus souvent les expropriés "à la barre" afin d'exercer une influence psychologique sur les jurés d'expropriation, habitués à considérer que lors des procès pénaux le suspect était à la barre et non au siège du ministère public. En l'absence de commissaire du gouvernement, «l'égalité des armes» se trouvait déjà rompue au bénéfice de l'administration expropriante $^{42}$. Sous ce rapport, on mesure qu'à travers la condamnation de la France dans l'affaire Yvon, c'est une certaine vision, assez autoritaire et «napoléonienne», du rapport de droit devant exister entre les expropriés et l'État qui a fait l'objet d'une remise en question.

Le caractère non contradictoire de l'ordonnance d'expropriation découlant de l'article L. 12-1 du Code de l'expropriation (ancien) ${ }^{43}$, qui constitue une autre caractéristique de la procédure d'expropriation issue directement de l'Empire ${ }^{44}$, a suscité des interrogations tant au regard de sa constitutionnalité ${ }^{45}$ que de sa conventionnalité, même si, à ce jour, aucune condamnation de la France n'a eu lieu par la Cour EDH. Dans son rapport pour l'année $2000^{46}$, la Cour de cassation s'était interrogée sur la compatibilité de ce principe avec les exigences du droit à un procès équitable. Mais, en 2001, elle avait en définitive jugé qu'il n'y avait pas de contradiction avec l'article $6, \S 1$, de la Convention EDH dans la mesure où le principe du contradictoire était respecté en amont de l'ordonnance d'expropriation, devant le juge administratif, et en aval, devant la Cour de cassation ${ }^{47}$. Même si cette configuration n'a aujourd'hui pas encore été contestée par la Cour EDH, cette résurgence de la loi du 8 mars 1810 pose question et ce, d'autant plus qu'au $\mathrm{XIX}^{\mathrm{e}}$ siècle, on admettait que les expropriés puissent fournir au procureur du roi leurs observations par des notes avant que soit prononcé le transfert de propriété ${ }^{48}$, afin de compenser l'impossibilité pour les expropriés de s'expliquer contradictoirement devant le juge ${ }^{49}$, ce qui signifie qu'à une époque où le «droit au procès équitable» n'était pas aussi présent qu'aujourd'hui dans le discours juridique - fût-ce sous un autre vocable - ni dans les normes en vigueur, le caractère non contradictoire de l'ordonnance d'expropriation avait déjà été perçu comme posant un problème au regard des garanties des expropriés. Les expropriés sont donc aujourd'hui, sur ce point, moins protégés juridiquement qu'ils ne l'étaient il y a un siècle, ce qui atteste combien l' «esprit napoléonien » est prégnant. Peut-on, dans ces conditions, envisager une condamnation de la France par la Cour EDH? Rien n'est moins sûr dans la mesure où celle-ci a déjà attesté qu'elle refusait d'analyser les procédures isolément au profit d'une lecture "globale», jugeant, à propos d'une affaire dans laquelle les requérants n'avaient pas été en mesure de contester en temps utile une DUP parce qu'ils n'avaient été informés que leur propriété était concernée par une opération qu'après la clôture du délai de recours contre

41. T. Ducrocq, «Des expropriants, du droit de poursuite appartenant à chacun d'eux, et de la place qu'ils doivent occuper à l'audience du jury d'expropriation", Revue critique de législation et de jurisprudence, 1866, p. 491-526.

42. Citons T. Ducrocq: «Nous estimons trop les citoyens désignés [...] pour remplir la haute mission de jurés en matière d'expropriation, pour supposer que leurs intelligences et leurs consciences puissent être plus sensibles aux paroles qui tomberaient du siège du ministère public, qu'aux bonnes raisons que la même voix leur adresserait de la barre. Mais si tel est notre sentiment personnel, nous avons, en sens contraire, entendu des expropriés manifester des appréhensions; et, parmi les expropriants, il paraît notamment que certaines compagnies de chemins de fer [...] ne partagent pas notre manière de voir, puisqu'elles demandent avec insistance à occuper devant le jury d'expropriation le siège du ministère public. Nous ne saurions admettre en effet qu'une telle demande pût avoir pour mobile exclusif un sentiment de vanité mal placée, sans une arrière-pensée d'intérêt plus sérieux et plus grave» (ibid., p. 494-495).

43. La réforme du nouveau Code de l'expropriation n'a pas modifié le principe selon lequel l'ordonnance est prononcée sans contradiction (voir art. L. 221-1 du nouveau Code). Voir S. Gilbert, «Réforme du Code de l'expropriation. Une codification “à droit constant” », La semaine juridique, éd. $G, \mathrm{n}^{\circ} 7,16$ février 2015,178 , p. 289-292.

44. Thomas-Philibert Ribout indiquait le 8 mars 1810, devant le corps législatif: «Ne cédons pas à la première impression que peut produire la célérité d'un jugement rendu sans la présence du propriétaire et sans la fixation préalable de l'indemnité. Considérons qu'il s'agit en ce moment de procurer l'exécution de l'article $545 \mathrm{du}$ Code civil, qui confère un privilège en faveur de l'utilité publique; que ce premier jugement est rendu sommairement et par défaut; que le propriétaire condamné peut recourir, et qu'ainsi ses droits sont encore entiers » (cité par C. de Lalleau, Traité de l'expropriation pour cause d'utilité publique, Bruxelles, Librairie de jurisprudence Tarlier, 1835, p. 192).

45. Saisi d'une QPC, le Conseil constitutionnel a déclaré l'ancien article L. 12-1 du Code de l'expropriation comme étant juridiquement conforme. Voir CC, déc. n 2012-247 QPC du 16 mai 2012, Consorts Lazaro: L'actualité juridique. Droit immobilier, 2012, p. 767, note S. Gilbert.

46. Cour de cassation, Rapport 200o: la protection de la personne, Paris, La documentation française, 2001 , p. 12 sq.: «on peut se demander si le caractère non contradictoire, à ce stade de la procédure, du transfert de propriété, est conforme aux exigences de l'article $6 \$ 1^{\text {er }}$ de la Convention de sauvegarde des droits de l'homme».

47. Cass., $3^{\mathrm{e}}$ civ., 12 décembre 2001, M. Guy Hain c. État: L'actualité juridique. Droit immobilier, 2002, p. 144, note R. Hostiou.

48. Voir A. Herson, De l'expropriation pour cause d'utilité publique ou commentaire de la loi du 3 mai 1841, Paris, Comptoir des imprimeurs unis, 1843 , p. 515

49. Voir C. Peyronny, E. Delamarre, Commentaire théorique et pratique des lois d'expropriation pour cause d'utilité publique, Paris, Marescq aîné, 1859 , p. 194, $\$ 222$ : «Les expropriés ne sont pas des parties». A. Herson indique en 1843: «Le procureur du Roi entendu, les parties intéressées peuvent présenter leurs observations. Toutefois, elles ne reçoivent pas d'assignation à comparaître, et si elles ont la faculté d'intervenir dans l'instance pour faire valoir leurs réclamations, la jurisprudence ne reconnaît, pas plus que la loi, l'obligation de les y rappeler» (De l'expropriation..., p. 65-66). 
le décret déclarant l'utilité publique, que s'ils avaient «été privés de l'opportunité de bénéficier de cette voie procédurale pour obtenir un contrôle juridictionnel du fondement de l'expropriation dont ils ont fait l'objet»,

[...] les requérants avaient également la possibilité, dans le cadre de leur recours contre l'arrêté de cessibilité, de soulever par voie d'exception l'illégalité du décret du 17 mars 1995 portant déclaration d'utilité publique et d'obtenir ainsi un contrôle juridictionnel de l'acte fondant l'expropriation litigieuse; par cette voie, ils auraient pu parvenir à l'annulation de cet arrêté sur le fondement de l'illégalité dudit décret, ce qui aurait fait obstacle au transfert de propriété ${ }^{\circ}$.

Le principe du dualisme procédural de la procédure d'expropriation suscite également de nombreuses interrogations s'agissant de sa conventionnalité. Le dualisme de la procédure d'expropriation est régulièrement remis en question ou, du moins, critiqué, au regard des inconvénients qu'il suscite. Pellegrino Rossi écrivait déjà, en 1851:

Nous avons $[\ldots]$ une procédure relative à l'expropriation des immeubles, qui est un de ces dédales, un de ces labyrinthes où il n'y a pas de raison pour que la justice se retrouve jamais ${ }^{51}$.

Ce caractère dual de la procédure constitue un facteur de télescopages de compétences juridictionnelles, ainsi qu'en atteste le contentieux de la rétrocession ${ }^{52}$, celui de l'indemnisation ou encore celui de la responsabilité ${ }^{53}$. Indifférente au dualisme juridictionnel, la Cour de Strasbourg vérifie que les inconvénients y afférant sont ou non constitutifs d'une violation de l'article $6, \$ 1$, de la Convention EDH. Sur ce fondement, la France a été condamnée, notamment ${ }^{54}$ dans l'affaire Guillemin (précitée), dans la mesure où «la procédure d'expropriation, [qui] présente une relative complexité, notamment en ce qu'elle relève de la compétence des deux ordres de juridiction ${ }^{55}$, n'avait pas permis que l'affaire fût jugée dans un délai raisonnable. La complexité des règles de compétence juridictionnelle peut également constituer une violation du droit européen au regard de la méconnaissance de l'article $1^{\mathrm{er}}$ du premier Protocole additionnel à la Convention EDH. Dans l'affaire Maupas et autres c. France (précitée), la Cour de Strasbourg a ainsi souligné, le 19 septembre 2006, à propos du contentieux de l'expropriation, que le non-respect de l'article $1^{\mathrm{er}} \mathrm{du}$ premier Protocole pouvait être lié à la mise en œuvre des procédures juridictionnelles. Même si, en l'espèce, la France n'a pas été condamnée - c'est, dans cette affaire, la possibilité d'invoquer l'illégalité de la déclaration d'utilité publique par voie d'exception qui a conduit la Cour à ne pas constater de violation de l'article $1^{\text {er }} \mathrm{du}$ premier Protocole -, l'architecture du contentieux de l'expropriation était toutefois en question.

S'agissant des difficultés d'articulation entre la phase judiciaire et la phase administrative, la loi no 95-101 du 2 février 1995, dite loi Barnier, a permis (art. L. 12-5, al. 2, du Code de l'expropriation) de faire constater par le juge de l'expropriation que l'ordonnance était dépourvue de base légale lorsqu'un élément de la phase administrative était définitivement annulé, ce qui a représenté une amélioration des garanties accordées aux expropriés au regard de l'état du droit antérieur. Le décret no 2005-467 du 13 mai $2005^{56}$ a ensuite modifié l'articulation des deux phases susévoquées (art. R. 12-2-1 du Code de l'expropriation) sous l'angle $\mathrm{du}$ «droit au procès équitable» en prévoyant que si la déclaration d'utilité publique ou l'arrêté de cessibilité sont suspendus dans le cadre d'un recours en référé-suspension, le préfet, dès réception de la notification de la décision de suspension, en informe le juge de l'expropriation qui sursoit au prononcé de l'ordonnance d'expropriation dans l'attente d'une décision définitive. Une vision plus «horizontale» de la procédure d'expropriation s'est donc imposée mais des difficultés demeuraient, à la suite de la réforme du 13 mai 2005; il était notamment possible que le préfet ignorât l'existence d'un recours si la déclaration d'utilité publique était prise par un arrêté ministériel ou par un décret en Conseil d'État. La réforme du Code de l'expropriation survenue à la fin de l'année $2014^{57}$ a apporté divers aménagements qui tendent à résorber davantage qu'auparavant les inconvénients inhérents à l'écartèlement de la procédure d'expropriation entre les deux ordres de juridiction. De ce point de vue, la réforme en question contribue notamment à ce que le droit français de l'expropriation soit à l'abri d'une condamnation de la France par la Cour EDH.

Au stade du prononcé de l'ordonnance d'expropriation, il est désormais prévu par l'article R. 221-5 du Code de l'expropriation - en complément de l'article R. 221-3 reprenant l'ancien article R. 12-2-1 aux termes duquel « Si l'acte déclarant l'utilité publique, l'arrêté de cessibilité ou l'acte en tenant lieu fait l'objet d'une suspension dans le cadre d'une procédure de référé, le préfet en informe le juge dès qu'il a reçu notification de la suspension. Celui-ci sursoit au prononcé de l'ordonnance d'expropriation dans l'attente de la décision de la juridiction administrative

50. Cour EDH, 19 septembre 2006, Maupas et autres c. France, $\mathrm{n}^{\circ}$ 13844/02, \$ 21; L'actualité juridique. Droit administratif, 2007, p. 180, note R. Hostiou.

51. P. Rossi, Cours d'économie politique, t. III, De la distribution de la richesse, Paris, G. Thorel, 1851, p. 303.

52. Voir S. Gilbert, «La responsabilité de l'expropriant dans le cadre de la violation du droit de rétrocession de l'article L. 12-6 du Code de l'expropriation", Droit et Ville, 2000, p. 143-174.

53. Voir R. Hostiou, «La juridiction administrative et le contentieux de la responsabilité de la puissance publique en matière d'expropriation pour cause d'utilité publique", in Mouvement du droit public: du droit administratif au droit constitutionnel. Mélanges en l'honneur de Franck Moderne, Paris, Dalloz, 2004, p. 219-232.

54. Voir aussi Cour EDH, 16 décembre 1992, De Geouffre de La Pradelle c. France, n 12964/87.

55. Cour EDH, 21 février 1997, Guillemin c. France, $\$ 42$.

56. Voir R. Hostiou, «L'expropriation aux normes européennes», Études foncières, n 115, mai-juin 2005, p. 7-12.

57. Ordonnance $\mathrm{n}^{\circ}$ 2014-1345 du 6 novembre 2014 (JORF, $\mathrm{n}^{\circ}$ 261, 11 novembre 2014, p. 19003) et décret n $2014-1635$ du 26 décembre 2014 (JORF, $n^{\circ} 300,28$ décembre 2014, p. 22598); R. Hostiou, «Le Code de l'expropriation pour cause d'utilité publique, version 2015. Un coup pour (presque) rien?", L'actualité juridique. Droit administratif, 2015, p. 689. 
sur le fond de la demande» - que le juge de l'expropriation refuse de prononcer l'ordonnance d'expropriation, notamment si «la déclaration d'utilité publique ou les arrêtés de cessibilité sont caducs ou ont été annulés par une décision définitive du juge administratif». Avant la réforme du Code, l'article R. 12-3 ne prévoyait une telle obligation que si «le dossier n'est pas constitué conformément aux prescriptions de l'article R. 12-1, ou si la déclaration d'utilité publique ou les arrêtés de cessibilité sont caducs ». Cette interdiction faite au juge de l'expropriation de prononcer le transfert de propriété en cas d'annulation de la DUP ou de l'arrêté de cessibilité était déjà sous-entendue avant la réforme, ce qui relativise, de ce point de vue, l'apport de l'article R. 221-5, mais ce qui va sans dire va mieux en se disant. S'agissant de la possibilité de faire constater le défaut de base légale de l'ordonnance d'expropriation lorsqu'un élément de la phase administrative (DUP ou arrêté de cessibilité) a été définitivement annulé, une évolution assez significative est opérée.

Si l'article R. 223-2, alinéa $1^{\mathrm{er}}$, prévoit qu'

À peine d'irrecevabilité de sa demande tendant à faire constater le manque de base légale de l'ordonnance d'expropriation, l'exproprié saisit le juge dans un délai de deux mois à compter de la notification de la décision définitive du juge administratif annulant la déclaration d'utilité publique ou l'arrêté de cessibilité.

Ce qui est conforme à l'ancien article R. 12-5-1 - l'alinéa 2 dudit article dispose que «Si l'exproprié n'a pas été partie à la procédure devant la juridiction administrative, le délai de deux mois court à compter de la réception de la lettre d'information prévue à l'article R. 223-3", l'article R. 223-3 disposant quant à lui, de manière inédite, que:

Pour les expropriés qui n'avaient pas la qualité de partie à l'instance devant le juge administratif ayant décidé l'annulation de la déclaration d'utilité publique ou de l'arrêté de cessibilité, le délai de deux mois mentionné à l'article R. 223-2 ne court qu'à compter de la date à laquelle ils sont informés de cette annulation par l'expropriant. L'information incombant à l'expropriant est faite par lettre recommandée avec demande d'avis de réception ou par voie de signification.

Ce nouveau mécanisme semble au premier abord plus protecteur des expropriés que ce que prévoyait l'ancien dispositif issu du décret de 2005. Mais il est aussi possible que le délai de deux mois susvisé courre tardivement si l'expropriant tarde à informer l'exproprié qui n'était pas partie à l'instance et ce, alors même que la prise de possession aurait eu lieu et que l'affectation du bien confor- mément à la destination prévue dans la DUP aurait été en partie réalisée. En cas d'annulation par une décision définitive du juge administratif de la DUP ou de l'arrêté de cessibilité, il est désormais prévu par l'article L. 223-2 que:

Sans préjudice de l'article L. 223-1, en cas d'annulation par une décision définitive du juge administratif de la déclaration d'utilité publique ou de l'arrêté de cessibilité, tout exproprié peut faire constater par le juge que l'ordonnance portant transfert de propriété est dépourvue de base légale et demander son annulation.

Après avoir constaté l'absence de base légale de l'ordonnance portant transfert de propriété, le juge statue sur les conséquences de son annulation.

C'est, à ce stade, la première fois qu'il est possible de demander au juge de l'expropriation d'annuler l'ordonnance d'expropriation, en sus du constat de son manque de base légale, même si, sur le plan pratique, les effets de ces deux demandes sont équivalents, pour ne pas dire redondants. Cette réforme, en ce qu'elle tend à résorber les inconvénients inhérents au dualisme juridictionnel, contribue à prémunir la France d'une condamnation par la Cour EDH, ce qui, en creux, atteste l'influence du droit européen sur le régime juridique de l'expropriation en droit interne.

S'agissant désormais du droit de rétrocession ${ }^{58}$, qui constitue pour les anciens propriétaires expropriés une garantie très ancienne - il a été introduit dans l'ordonnancement juridique par la loi du 7 juillet 1833 (art. 6o) sous le nom de «droit de remise » - contre l'absence d'affectation conforme à la DUP des biens expropriés, la question se pose de savoir si la France pourrait faire l'objet d'une condamnation par la Cour EDH car cette garantie peut être très facilement paralysée alors même qu'aucune affectation conforme à la DUP n'aurait été réalisée. En effet, rappelons que l'intervention d'une nouvelle DUP ou la prorogation de la DUP initiale empêchent le juge judiciaire de droit commun de reconnaître le droit de rétrocession, sauf s'il apparaît, dans le prolongement de la jurisprudence Motais de Narbonne (précitée) relative aux réserves foncières, que l'ancien propriétaire a été privé d'une plus-value qui aurait été engendrée par le bien exproprié et qu'il a «subi une charge excessive du fait de l'expropriation ${ }^{59}$. Pour paralyser une demande de rétrocession, la «nouvelle» DUP doit certes concerner les mêmes parcelles que celles qui ont été expropriées $^{60}$, ne pas révéler de détournement de procédure $^{61}$ ni de détournement de pouvoir ${ }^{62}$. Mais dès lors que ces exigences sont satisfaites, cette «arme» en possession de l'expropriant se révèle d'autant plus redoutable qu'elle peut être utilisée sans limitation de nombre ou de temps ${ }^{63}$.

58. Voir S. Gilbert, «Le droit de rétrocession des biens expropriés», Opérations immobilières, mars 2013, p. 27-31.

59. Cass., $3^{\mathrm{e}}$ civ., 19 novembre 2008, M. Payet c. commune du Tampon, $\mathrm{n}^{\mathrm{o}}$ 07-15705, Bulletin civil III, $\mathrm{n}^{\circ} 176:$ L'actualité juridique. Droit immobilier, 2009, p. 312, note R. Hostiou.

60. Cass., $3^{\mathrm{e}}$ civ., 17 mars 1999, Homberg, $\mathrm{n}^{\circ}$ 98-14751.

61. CE, 8 novembre 2000, Époux Norais, nº 176394: L'actualité juridique. Droit immobilier, 2001, p. 355.

62. À propos d'une réserve foncière constituée pour tenir en échec une demande de rétrocession: CE, 12 mai 2004, Département des Alpes-Maritimes, $\mathrm{n}^{\circ}$ 253586: La semaine juridique, édition administrations et collectivités territoriales, $\mathrm{n}^{\circ} 22,24$ mai 2004, p. 1575.

63. Cass., $3^{\mathrm{e}}$ civ., 10 avril 1996, Commune de Gillancourt, $\mathrm{n}^{\circ}$ 94-15761. 
Si, en droit européen, l'expropriation doit être justifiée par une «cause d'utilité publique» (art. ${ }^{\text {er }}$ du premier Protocole additionnel à la Convention EDH), il existe un risque qu'une expropriation dépourvue d'affectation conforme puisse être considérée comme méconnaissant cet article et que l'article L. 421-1 du Code de l'expropriation relatif au droit de rétrocession soit considéré comme étant juridiquement non conforme. Ce risque est-il significatif? Il ne le semble pas, car s'il est très aisé d'empêcher que soit reconnu le droit de rétrocession (voir supra), il s'avère que tant que le droit de rétrocession n'est pas reconnu, il n'y a en principe pas d'atteinte à un «bien" au sens de l'article $1^{\text {er }}$ du premier Protocole additionnel. La Cour EDH a décidé en ce sens que:

[...] le tribunal d'arrondissement n'investissait pas les requérants d'un droit exécutoire à obtenir la rétrocession des terrains en cause [...] et n'avait fait naître dans le chef de ceux-ci une créance suffisamment établie pour s'analyser en une "espérance légitime» au sens de la jurisprudence de la Cour.

Dans ces conditions, la Cour estime que dans le contexte de leur demande de rétrocession les requérants n'avaient pas un «bien» au sens de la première phrase de l'article 1 du Protocole $n^{\circ} 1^{64}$.

La Cour EDH a cependant jugé qu'un refus de rétrocession n'était pas justifié au regard de l'article $1^{\mathrm{er}} \mathrm{du}$ premier Protocole additionnel de la Convention EDH, dans l'hypothèse où un bien n'est pas affecté conformément à une DUP au bout d'un laps de temps important:

Si l'on peut [...] aisément accepter que les exigences de l'intérêt public justifi[ent] l'expropriation [...], la Cour ne considère pas justifié le maintien de la décision de non-restitution des biens, eu égard [notamment] au fait que les terrains litigieux n'ont toujours pas été affectés à la réalisation d'ouvrages d'intérêt public ${ }^{65}$.

Il n'existe cependant pas de procédure correspondant à un droit de rétrocession dans le droit interne de Saint-Marin, de sorte qu'il est probable que la portée de cette jurisprudence ne soit pas «transposable» à la France en raison du caractère subsidiaire de la protection des droits et libertés assurée par la Cour $\mathrm{EDH}^{66}$. À l'issue de ce paragraphe, on observe que si la conventionnalité de certains aspects de la procédure d'expropriation suscite des interrogations, peu de caractéristiques de la procédure ont été modifiées pour tirer les conséquences de décisions de la Cour EDH, soit parce que les condamnations ont été rares, soit parce que les réformes de ladite procédure ont été menées le cas échéant pour anticiper d'éventuelles condamnations des juges strasbourgeois de sorte que, dans ce cas, l'influence de la Cour EDH sur le droit français de l'expropriation n'est, au mieux, qu'indirecte. Examinons maintenant la conventionnalité des principes relatifs au droit et à l'évaluation de l'indemnité d'expropriation.

\section{B. La conventionnalité des principes relatifs au droit et à l'évaluation de l'indemnité d'expropriation}

L'étude des principes relatifs au droit et à l'évaluation de l'indemnité d'expropriation porte sur un champ immense de sorte que, dans les pages qui suivent, on se contentera d'identifier et d'analyser des problèmes présentant un intérêt qui nous a semblé particulièrement vif, sans prétendre à l'exhaustivité. À titre liminaire, il convient de rappeler que, pour la Cour EDH, l'expropriation doit, selon une jurisprudence constante, ménager un «juste équilibre» entre les exigences de l'intérêt général de la communauté et les impératifs de sauvegarde des droits fondamentaux de l'individu, cet équilibre étant rompu si l'exproprié a dû subir une charge dite «spéciale et exorbitante». À ce titre, la Cour de Strasbourg contrôle si l'exproprié a bénéficié d'une indemnisation "raisonnablement en rapport avec la valeur du bien dont il a été privé ». Ce contrôle, réduit, se limite toutefois à déterminer si les modalités retenues excèdent "la large marge d'appréciation» dont bénéficient en la matière les autorités nationales ${ }^{67}$, en raison du caractère subsidiaire du système de protection des droits existant en la matière, ce qui donne à penser que les condamnations de la France relatives au droit ou aux principes d'indemnisation des biens expropriés seront rares sinon très rares.

Parmi les problèmes les plus intéressants que pose la jurisprudence de la Cour EDH en matière d'indemnisation des biens expropriés, relevons en premier lieu qu'en vertu de la décision Lallement c. France (précitée), il convient désormais d'indemniser l'outil de travail que peut représenter le terrain exproprié lorsque l'exproprié, propriétaire du bien, utilise celui-ci comme son outil de travail. Une telle solution, en décalage avec les principes du droit français de l'expropriation, car beaucoup plus

64. Cour EDH, 24 avril 2008, Kemp et autres c. Luxembourg, $\mathrm{n}^{\circ}$ 17140/05, $\$ 76-77$.

65. Cour EDH, 13 juillet 2004, Beneficio Cappella Paolini c. Saint-Marin, no 40786/98, \$33.

66. À défaut de rétrocession, les anciens propriétaires peuvent aujourd'hui, dans certains cas, espérer une indemnisation au motif qu'en l'absence d'affectation effective des biens expropriés en vue de la création d'une réserve foncière, ils sont en droit de se voir allouer l'intégralité de la plusvalue dont lesdits biens ont bénéficié depuis leur acquisition par les autorités publiques (Cour EDH, 2 juillet 2002, Motais de Narbonne). La Cour de cassation, dans le prolongement de cette jurisprudence, a considéré qu'en se contentant, pour débouter les expropriés de leur demande de rétrocession, de constater l'existence d'une DUP «en cours de validité», sans rechercher, comme cela lui était demandé, si ceux-ci n’avaient pas été indûment privés d'une plus-value et s'ils n'avaient pas en conséquence subi une charge excessive du fait de l'expropriation, une cour d'appel n'a pas «donné de base légale à sa décision» (Cass., $3^{e}$ civ., 19 novembre 2008, M. Payet c. commune du Tampon: L'actualité juridique. Droit immobilier, 2009, p. 312, note R. Hostiou). Voir S. Gilbert, "L'indemnisation de la perte de plus-value du bien exproprié "hors rétrocession" ", L'actualité juridique. Droit administratif, 2016, p. 58-63.

67. Cour EDH, 4 novembre 2010, Dervaux c. France, nº 40975/07: L'actualité juridique. Droit administratif, 2010, p. 2493, note R. Hostiou. 
protectrice des expropriés, représente une avancée majeure de la jurisprudence de la Cour $\mathrm{EDH}^{68}$.

En deuxième lieu se pose la question de l'indemnisation du préjudice moral. Alors que ce préjudice n'est pas indemnisé en droit français, peut-il l'être en vertu du droit européen? Le Code de l'expropriation prévoit que «les indemnités allouées doivent couvrir l'intégralité du préjudice direct, matériel et certain, causé par l'expropriation» (art. L. 321-1 du nouveau Code; art. L. 13-13 de l'ancien Code). Le 21 octobre 2010 ( $\operatorname{arrêt}^{\circ}{ }^{\circ} 1348$ ), la Cour de cassation a transmis au Conseil constitutionnel une QPC visant à déterminer si cet article, en tant qu'il limite l'indemnité d'expropriation au préjudice matériel, à l'exclusion du préjudice moral, était conforme à l'article 17 de la Déclaration de 1789. Avant d'être saisi de cette question, le Conseil constitutionnel avait déjà jugé que «pour être juste, l'indemnisation doit couvrir l'intégralité du préjudice, direct, matériel et certain, causé par l'expropriation ${ }^{69}$. Si la décision $n^{\circ}$ 2010-87 QPC du 21 janvier 2011 reproduit in extenso cette solution, c'est en revanche la première fois qu'a été examinée la question de savoir si l'absence d'indemnisation du préjudice moral consécutif à l'expropriation était constitutionnelle. Le Conseil constitutionnel a décidé que:

Aucune exigence constitutionnelle n'impose que la collectivité expropriante, poursuivant un but d'utilité publique, soit tenue de réparer la douleur morale éprouvée par le propriétaire à raison de la perte des biens expropriés; que, par suite, l'exclusion de la réparation du préjudice moral ne méconnaît pas la règle du caractère juste de l'indemnisation de l'expropriation pour cause d'utilité publique ${ }^{70}$.

Si la «motivation» formulée par le Conseil constitutionnel ne constitue nullement une argumentation, il est probable que nous soyons en présence d'une loi certes valide mais pour autant non conforme à l'article 17 de la Déclaration de 1789. Car si le prix représente la compensation du vendeur, il ne l'est pas pour l'exproprié qui, à ce titre se voit accorder une «indemnité» et non un "prix». Or, comment admettre qu'une indemnité ne couvrant pas tous les préjudices, c'est-à-dire une indemnité qui n'est pas complète, puisse être juste? Il y a là une contradiction patente révélatrice d'une conception très «napoléonienne» du degré de protection qu'il est convenu d'accorder à l'exproprié. La conception que développe le Conseil constitutionnel (en refusant d'indemniser le préjudice moral consécutif à l'expropriation alors que l'article 17 de la Déclaration de 1789 prescrit toutefois l'octroi d'une «juste» indemnité) est tout droit héritée de la conception que développait autrefois l'Administration à ce sujet, puisque celle-ci refusait également aux expropriés la possibilité de recevoir une indemnité d'expropriation sur le fondement d'un préjudice moral. Cette conception très «napoléonienne» du respect qu'il convient d'accorder au droit de l'individu exproprié a été très abondamment critiquée par la doctrine civiliste du $\mathrm{XIX}^{\mathrm{e}}$ siècle ${ }^{71}$. Et parce qu'un consensus s'était dégagé à ce sujet à cette époque, le préjudice moral était alors indemnisé de manière régulière ${ }^{72}$, avec le soutien majoritaire de la doctrine ${ }^{73}$, bien que l'Administration tente de s'y opposer avec constance ${ }^{74}$. Compte tenu de la décision du Conseil constitutionnel susvisée en date du 21 janvier 2011, le juge de l'expropriation peut ainsi continuer de refuser d'indemniser le préjudice résultant de l'attachement particulier de l'exproprié à un bien familial ${ }^{75}$, de la rupture d'une unité familiale ${ }^{76}$, d'une perte d'agrément ${ }^{77}$, de la disparition d'avantages divers liés à l'usage du bien faisant l'objet de l'expropriation $^{78}$ ou de la situation particulière de l'exproprié ${ }^{79}$.

68. Dans le prolongement de cette jurisprudence, la Cour EDH a admis, dans un contentieux qui concernait l'Italie et l'absence d'indemnisation d'un bail commercial, que le locataire d'un terrain subissait une charge spéciale et exorbitante contraire au droit au respect de ses biens s'il n'obtenait pas une indemnisation raisonnablement et concrètement en rapport avec le préjudice subi du fait de l'expropriation. En l'espèce, l'indemnisation qui lui avait été octroyée ne prenait pas en compte la perte de l'outil de travail (Cour EDH, 26 avril 2001, Di Marco c. Italie, $\mathrm{n}^{\circ} 32521 / 05$ ).

69. CC, déc. n 89-256 DC du 25 juillet 1989, cons. 19; CC, déc. nº 2010-26 QPC du 17 septembre 2010, cons. 6.

70. R. Hostiou, «L'indemnité d'expropriation est-elle “juste” ? , L'actualité juridique. Droit administratif, 2011, p. 447.

71. Louis-Jean-Joseph Cappeau, président de la cour royale d'Aix, écrit ainsi en 1825: «Qui dit indemnité, ne dit ni prix ni valeur vénale seulement, ni moins encore juste prix, mais dédommagement de la privation: ce dédommagement ne peut être complet, qu'autant qu'on trouve, dans la somme qui le compose, et la valeur vénale et le prix d'affection, de convenance, d'agrément ou de commodité qui quelquefois est tout pour le propriétaire, et qui n'entre point dans la valeur vénale, parce qu'il manque de réalité pour le commun des acheteurs » (Traité de la législation rurale et forestière, Marseille, A. Ricard, 1825, t. III, p. 220-221, ici p. 220).

72. Voir, par exemple, Cass., 19 décembre 1838, Préfet de Seine-et-Oise c. Chemin de fer de Versailles, Recueil Sirey, 1839, 1, 30; Recueil Sirey, 1839, 1, 255.

73. Ferdinand Debray écrit, à propos des offres d'indemnité de l'expropriant au propriétaire, que «ces offres [...] doivent être de la véritable valeur des biens, et même quelque chose en sus: car, à moins que les travaux à exécuter n'ajoutent de la valeur aux propriétés, cas qui sera examiné dans le cours de cet ouvrage, il faut tenir compte de l'affection qu'on peut avoir pour les choses, et du dérangement dans les habitudes établies» (Manuel de l'expropriation pour cause d'utilité publique, Paris, A. Durand, 1845, p. 46-47).

74. Caudaveine et Théry rapportent en 1839 que " “constamment [indiquait un député non identifié, en 1833], l'administration a contesté l'étendue et la définition du mot indemnité; elle a soutenu devant les Tribunaux que l'indemnité qui était due n'était que la valeur vénale du sol [...]; prétentions contre lesquelles les Tribunaux se sont toujours élevés, parce qu'ils ont justement et sagement interprété les dispositions de l'article 545 du Code civil, parce qu'ils ont pensé que l'indemnité, pour être juste, devait être complète. En effet, le mot indemnité ne veut pas dire seulement prix vénal de l'immeuble; il veut dire aussi le dédommagement dû au propriétaire par suite de sa dépossession. [...]" [...] ce qu'il faut entendre par indemnité, ce n'est pas la valeur vénale du fonds exproprié, c'est la réparation du dommage souffert, soit d'une manière absolue, soit d'une manière relative, par ceux qui exercent des droits quelconques sur cette propriété» (Traité de l'expropriation pour cause d'utilité publique, Paris, A. Guyot et Scribe, 1839, p. 205-206).

75. Cass., $3^{\mathrm{e}}$ civ., 30 mai 1972, Consorts Bourgeois, Bulletin civil III, $\mathrm{n}^{\circ} 225$.

76. Cass., ch. temp. expr., 29 octobre 1965, Dame Sicart c. ville de Paris, Bulletin civil V, n 118, p. 96.

77. Cass., $3^{\mathrm{e}}$ civ., 3 décembre 1970, Bulletin civil III, $\mathrm{n}^{\circ} 487$.

78. CA Rouen, 18 juin 1968, J. de Meunynck: Gazette du Palais, 1968, 2, 336.

79. Cass., $3^{\mathrm{e}}$ civ., 17 octobre 1972 , Laurier, Bulletin civil III, $\mathrm{n}^{\circ} 524$, p. 382. 
Peut-on obtenir de la Cour EDH une protection du droit de propriété qui soit supérieure à celle qui résulte de la décision $n^{\circ}$ 2010-87 QPC? Dans l'arrêt Lallement c. France, la Cour de Strasbourg a jugé qu'un éleveur exproprié d'une grande partie de sa propriété ayant

[...] été privé de son «outil de travail» sans compensation appropriée $[\ldots]$ [d]e telles circonstances sont sans aucun doute de nature à générer angoisse et tension [de sorte que] le requérant peut donc se prévaloir d'un préjudice moral justifiant l'octroi d'une indemnité ${ }^{80}$.

Le préjudice moral n'est donc pas dû à l'expropriation en elle-même mais à l'angoisse et la tension causées par l'absence de compensation appropriée. Il existe là un certain paradoxe: l'angoisse et la tension sont redevables à la fixation de l'indemnité d'expropriation et non à la privation de la propriété. Comment l'expliquer? L'absence de compensation appropriée crée une situation source d'angoisse et de tension dans la mesure où l'éleveur n'est pas, par voie de conséquence de l'octroi de l'indemnité d'expropriation, en mesure de poursuivre son activité. Ce n'est donc pas l'insuffisance ou l'incomplétude de l'indemnité qui est en elle-même source d'angoisse et de tension sinon les conséquences que celle-ci revêt. Il apparaît donc, aujourd'hui, que la Cour EDH n'assure pas une protection supérieure à celle du Conseil constitutionnel s'agissant de l'indemnisation du préjudice moral.

Évoquons, en troisième lieu, un récent arrêt rendu par la Cour EDH qui a reconnu le droit des expropriés à être indemnisés à hauteur de la «valeur marchande» de leur bien ${ }^{81}$. Une telle formulation est-elle de nature à faire naître des espoirs chez les expropriés qui éprouvent le sentiment que leur bien n'a pas été évalué conformément à sa valeur sur le marché ? Rappelons qu'en l'espèce un terrain situé dans le centre d'une ville, à proximité immédiate de la place principale de celle-ci, avait été classé comme étant une «zone agricole» par le plan d'urbanisme puis exproprié pour y construire un espace commercial. Les juges italiens avaient accordé à ce dernier une indemnité correspondant au rendement moyen des terres agricoles de la région où était situé ce bien en considérant que le bien devait être indemnisé en tant que terrain agricole utilisé en agriculture, et ce, alors même que le terrain devait être construit. La Cour EDH a dès lors conclu à la violation de l'article $1^{\text {er }}$ du premier Protocole additionnel à la Convention EDH, estimant que l'indemnisation accordée au requérant n'avait pas été adéquate, vu son faible montant et l'absence de raisons d'utilité publique pouvant légitimer une indemnisation tellement inférieure à la valeur marchande du bien. Si cet arrêt représente une nouvelle «fenêtre juridique» pour remettre en question des indemnités d'expropriation, observons cependant qu'il existe en droit interne certains dispositifs juridiques visant à empêcher que l'indemnité soit déconnectée de la «valeur marchande» du bien exproprié: d'une part, l'obligation de tenir compte des servitudes et restrictions administratives affectant le bien, donc du classement dudit bien au regard du plan d'urbanisme applicable, sous réserve qu'il n'existe pas d' « intention dolosive»; d'autre part, la notion de terrain bénéficiant d'une "situation hautement ou éminemment privilégiée », qui permet au juge de l'expropriation de tenir compte de la présence d'équipements divers, de réseaux de viabilité ou de voies de circulation conférant à un terrain qui n'est pas qualifié de «terrain à bâtir» un statut distinct de la terre à vocation exclusivement agricole ${ }^{82}$. Il ne semble donc pas que cet arrêt permette aisément d'obtenir une condamnation de la France de nature à ce que, le cas échéant, soit opérée une modification du Code de l'expropriation ou de la jurisprudence du juge judiciaire relative à la fixation des indemnités d'expropriation.

En dernier lieu, nous souhaitons insister sur un problème qui se pose régulièrement en matière d'indemnisation des biens expropriés et qui, contrairement aux autres hypothèses sus-évoquées, pourrait donner lieu à une éventuelle condamnation de la France par la Cour EDH. Il s'agit de l'impossible restitution d'un bien indûment exproprié lorsqu'il a été constaté par le juge de l'expropriation que l'ordonnance d'expropriation est dépourvue de base légale ou que l'ordonnance a été cassée pour ce même motif par la Cour de cassation. Dans une telle hypothèse, la Cour de cassation estime que le juge de l'expropriation ne peut ordonner la démolition d'un ouvrage public dès lors qu'il n'existe qu'une emprise irrégulière; aussi laisse-t-elle à l'exproprié la seule possibilité de demander, de ce fait, une indemnisation du préjudice résultant de la non-restitution du bien :

[...] le particulier irrégulièrement exproprié [...] qui ne peut bénéficier de la restitution [décide la Cour de cassation] doit recevoir la valeur réelle de l'immeuble au jour de la décision constatant l'absence de restitution sous la seule déduction de l'indemnité déjà perçue, augmentée des intérêts au taux légal ${ }^{83}$.

Quels sont, dans cette hypothèse, les préjudices indemnisables? Un arrêt de la Cour d'appel d'Aix-en-Provence, qui ne les identifie pas de façon nécessairement exhaustive, relève à ce sujet que:

[...] les dispositions de l'article R. 12-5-4 du code de l'expropriation [ancien] ${ }^{84}$ visent à indemniser les préjudices de jouissance ou d'exploitation liées à l'indisponibilité du bien exproprié, telle que l'impossibilité avérée de vendre le bien,

80. Cour EDH, 11 avril 2002, Lallement c. France, $\$ 31$.

81. Cour EDH, 17 novembre 2015, Preite c. Italie, $\mathrm{n}^{\circ}$ 28976/05: Revue de droit immobilier, 2016, p. 22, note R. Hostiou.

82. Cass., $3^{\text {e }}$ civ., $1^{\text {er }}$ décembre 1993, Consorts Vigoureux, $\mathrm{n}^{\circ}$ 92-70457.

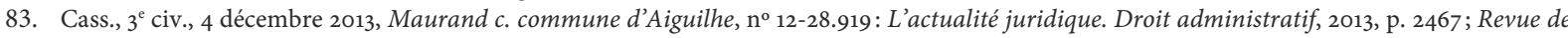
droit immobilier, 2014, p. 95, note R. Hostiou.

84. L'article R. 223-6 I du nouveau Code dispose uniquement que «si le bien exproprié n'est pas en état d'être restitué, l'action de l'exproprié se résout en dommages et intérêts". 
ou la perte de chance liée à l'impossibilité d'accomplir les actes nécessaires à la valorisation du bien dont il s'agit [...].

- ce qui semble très difficile à évaluer - et ajoute que le «préjudice moral» n'entre pas dans le cadre de l'article R. $12-5-4^{85}$. D'autres arrêts, dont les motifs sont un peu moins précis, relèvent que:

[...] l'expropriant, auteur d'une emprise irrégulière à la suite de l'annulation de son titre d'expropriation, est tenu d'indemniser l'exproprié de l'ensemble de son préjudice résultant des conséquences de l'emprise irrégulière $[\ldots]^{86}$.

La conformité de tels modes d'évaluation de l'indemnité due en cas de non-restitution d'un bien indûment exproprié pose question au regard de l'article $1^{\text {er }}$ du premier Protocole additionnel à la Convention EDH. La Cour EDH, dont la jurisprudence relative à cette question a évolué et est à cet égard plus précise que celle de la haute juridiction judiciaire, se révèle plus protectrice des victimes d'une expropriation indue. Elle a estimé, dans un premier temps, que l'expropriant devrait «assurer le paiement d'une somme correspondant à la valeur qu'aurait la restitution en nature», si elle était possible, et que l'expropriant devrait «allouer, s'il y a lieu, des dommages-intérêts pour les pertes subies et qui ne seraient pas couvertes par la restitution en nature ou le paiement qui en prend place ${ }^{87}$. À la faveur d'un revirement de jurisprudence ${ }^{88}$, la Cour EDH a dans un second temps décidé qu'en matière d'expropriation indirecte il ne devait plus être tenu compte, pour évaluer le dommage matériel, du coût de construction des immeubles bâtis sur les terrains, qu'il y avait lieu de prendre en considération la date à laquelle les intéressés ont eu la certitude juridique d'avoir perdu leur droit de propriété sur le bien litigieux, que la valeur vénale totale du bien fixée à cette date par les juridictions nationales était « ensuite à réévaluer et à majorer des intérêts au jour de l'adoption de la décision fixant l'indemnité», et que, du montant ainsi obtenu, serait «déduite la somme versée au requérant par les autorités de son pays ${ }^{89}$. Enfin, dans une autre affaire où il existait une expropriation indirecte, la Cour EDH a fixé un nou- veau mode d'évaluation y afférant qui s'applique en cas d'obstacle à la restitution des biens indûment expropriés. La Cour prévoit d'une part que:

L'indemnisation doit [...] correspondre à la valeur pleine et entière du terrain au moment de la perte de la propriété, telle qu'établie par l'expertise ordonnée par la juridiction compétente au cours de la procédure interne.

Qu'ensuite,

[...] une fois que l'on aura déduit la somme éventuellement octroyée au niveau national, ce montant doit être actualisé pour compenser les effets de l'inflation. Il convient aussi de l'assortir d'intérêts susceptibles de compenser, au moins en partie, le long laps de temps qui s'est écoulé depuis la dépossession des terrains ${ }^{9 \circ}$.

Et qu'il convient d' «évaluer la perte de chances subie à la suite de l'expropriation litigieuse » ce qui implique, à ce titre,

[...] de prendre en considération le préjudice découlant de l'indisponibilité du terrain pendant la période allant du début de l'occupation légitime [...] jusqu'au moment de la perte de propriété $[\ldots]^{91}$.

Elle décide d'autre part que si «le sentiment d'impuissance et de frustration face à la dépossession illégale de leur bien a causé [aux requérants] un préjudice moral important $»$, il $« \mathrm{y}$ a lieu de réparer de manière adéquate ${ }^{92}$. Au vu de cette décision de la Cour EDH, il semble que, pour se conformer pleinement au droit européen, la Cour de cassation devrait admettre que le préjudice moral soit, le cas échéant, indemnisé - bien que les critères définis par la Cour de Strasbourg soient d'un maniement délicat - mais, surtout, que les effets de l'inflation pour actualiser le montant de la somme due à l'exproprié soient pris en compte, une fois que le montant de l'indemnité d'expropriation versée initialement à l'exproprié a été déduit de la valeur du terrain fixée par le juge de l'expropriation lors de l'appréciation de la demande de restitution et d'indemnisation y afférant.

85. CA Aix-en-Provence, 4 avril 2013, RG: 10/00040.

86. CA Nîmes, $2^{\mathrm{e}}$ ch., 4 mai 2009, RG: 08/02936.

87. Cour EDH, 6 mars 2007, Scordino c. Italie, $\mathrm{n}^{\circ}$ 43662/98: Droit administratif, avril 2007, alerte $\mathrm{n}^{\circ} 10$, note R. Noguellou.

88. Cour EDH, 22 décembre 2009, Guiso-Gallisay c. Italie, $\mathrm{n}^{\circ} 58858$ /oo.

89. Ibid.

90. Cour EDH, 14 janvier 2014, Pascucci c. Italie, n $\mathrm{n}^{\mathrm{1}}$ 1537/04, $\$ 31$.

91. Ibid., $\$ 34$.

92. Ibid., $\$ 37$. 